\title{
Visualization of HIFU-induced lesions with thermoacoustic tomography
}

Xing Jin, Yuan Xu, Lihong V. Wang, Claudio I. Zanelli, Samuel M. Howard, et al.

Xing Jin, Yuan Xu, Lihong V. Wang, Claudio I. Zanelli, Samuel M. Howard, Yuncai Fang, "Visualization of HIFU-induced lesions with thermoacoustic tomography," Proc. SPIE 5320, Photons Plus Ultrasound: Imaging and Sensing, (12 July 2004); doi: 10.1117/12.532359

SPIE. Event: Biomedical Optics 2004, 2004, San Jose, CA, United States 


\title{
Visualization of HIFU-induced lesions with thermoacoustic tomography
}

\author{
Xing Jin, Yuan Xu and Lihong V. Wang* \\ Optical Imaging Laboratory, Department of Biomedical Engineering \\ Texas A\&M University, 3120 TAMU, College Station, Texas 77843-3120 \\ Claudio I. Zanelli, Samuel M. Howard and Yuncai Fang \\ Onda Corporation, Sunnyvale, California 94089
}

\begin{abstract}
High-intensity focused ultrasound (HIFU) has proved to be an effective minimally invasive surgical technology. In this study, we focus on the visualization of HIFU-induced lesions using microwave-induced thermoacoustic tomography (TAT). TAT has high spatial resolution, comparable with ultrasound imaging, and high contrast, which is induced by differences in the microwave absorption rates between tumor tissue and normal tissue. TAT can, in addition, differentiate tumors before and after treatment. A single, spherically focused transducer operating at a center frequency of approximately $4 \mathrm{MHZ}$ was used to generate the focused field. The lesion was generated in porcine muscle. A localtomography-type reconstruction algorithm was applied to reconstruct the TAT image of the lesions. The lesion shown by gross pathology confirms the corresponding region measured by TAT.
\end{abstract}

Keywords: Thermoacoustic tomography, High-intensity focused ultrasound

\section{INTRODUCTION}

High-intensity focused ultrasound has been used as an effective minimally invasive treatment of tumors deep in the body. The principle of HIFU is to use a highly focused ultrasound beam to destroy a predefined volume of malignant tissue, while leaving the surrounding tissue undamaged. The high-intensity ultrasound beam introduces a rapid rise in temperature for several seconds in the target region and thus results in tissue coagulation. This minimally invasive technique has been used to treat many tumors with only local aesthesia [1][2]. In China, HIFU has been used to treat thousands of patients for many types of cancer with very promising results.

Microwave-induced TAT uses a non-ionizing type of radiation. It combines the advantages of microwave imaging and ultrasonic imaging. When tissue absorbs pulsed microwave energy, it emits acoustic waves through thermo-elastic expansion. The thermoacoustic signals are then recorded to obtain the energy deposition of the tissue. Because of the short wavelength of the generated thermoacoustic waves, for example, in our case, the wavelength is about $1.5 \mathrm{~mm}$, TAT can obtain image resolution that is comparable with ultrasound imaging. Pure ultrasound imaging has good spatial resolution, but poor contrast for HIFU monitoring. The specific absorption rate (SAR) is a measure of the absorption of non-ionizing electromagnetic radiation by biological tissues. SAR can be expressed as $S A R=\sigma E^{2} / \rho$, where $\mathrm{E}$ is the electric field's intensity; $\sigma$ is the conductivity of the tissue and $\rho$ is the density of the tissue [4]. The conductivity of the tissue is related to its free water content, bound water content and ionic concentration. The deposition of microwave energy is largely decided by the free water content in the tissue. However, biological

\footnotetext{
* To whom all correspondence should be addressed. Telephone: 979-847-9040; Fax: 979-845-4450; Email: LWang@tamu.edu; URL: http://oilab.tamu.edu.
} 
tissue that has a large content of bound water can absorb more microwave energy than tissue with water in a free state [6]. The image contrast of TAT is brought about by the differences in the microwave absorption of the different tissues. The water content and ion constitute in the tissue affect its electromagnetic properties, and thus its ability to absorb microwave energy at different frequencies. Pure microwave imaging has poor imaging resolution because of its long wavelengths. Tissue thermal coagulation is associated with a decrease in the bound water and free water contents. Because TAT can differentiate thermal induced lesions from normal tissue based on differences in their electromagnetic properties, it can be used to image HIFU induced lesions. The purpose of this paper is to present some preliminary results of our research on using TAT to monitor HIFU-induced lesions.

\section{METHODS}

Figure 1 shows the side view of our experimental setup. The sample was made by embedding a piece of fresh porcine muscle in fat. The whole sample was immersed in mineral oil and placed on a base in the X-Y plane. The porcine muscle had a water content of $\sim 75 \%$ [3], which is similar to the water content of cancerous tissue; thus, in our experiments, it was used to simulate cancerous tissue.

The receiver was an unfocused transducer (V323, Panametric), with a central frequency of $2.25 \mathrm{MHz}$ and a diameter of $6 \mathrm{~mm}$. It rotated around the rotation axis in the X-Y plane. The center of the receiver was aligned with the center of the muscle. Thermal lesions were induced in the muscle using a spherically focused transducer (Onda corp.). The HIFU transducer operated at a central frequency of approximately $4 \mathrm{MHz}$, with a focal length of one inch. In the experiments, the HIFU transducer was immersed in mineral oil to provide coupling between the transducer and the muscle sample.

Pulsed microwave radiation was used to irradiate the samples. The microwave pulses were delivered to the sample through a horn waveguide with an opening of $120 \mathrm{~mm} \times 88 \mathrm{~mm}$. The frequency of the microwave was $3 \mathrm{GHz}$. At this frequency, the penetration depth in fat was $9 \mathrm{~cm}$ and the penetration depth in muscle was $1.2 \mathrm{~cm}$. Most other soft tissues have penetration depths between these two values. The microwave pulse width was $0.5 \mu$ s. Such short pulses can generate ultrasonic waves up to $2 \mathrm{MHz}$, thus providing spatial resolution at the millimeter level. The peak power of the microwave pulse was estimated to be $2 \mathrm{~kW}$, and the estimated total energy of the microwave pulse was about $10 \mathrm{~mJ}$. The microwave radiation level was below the safety requirement.

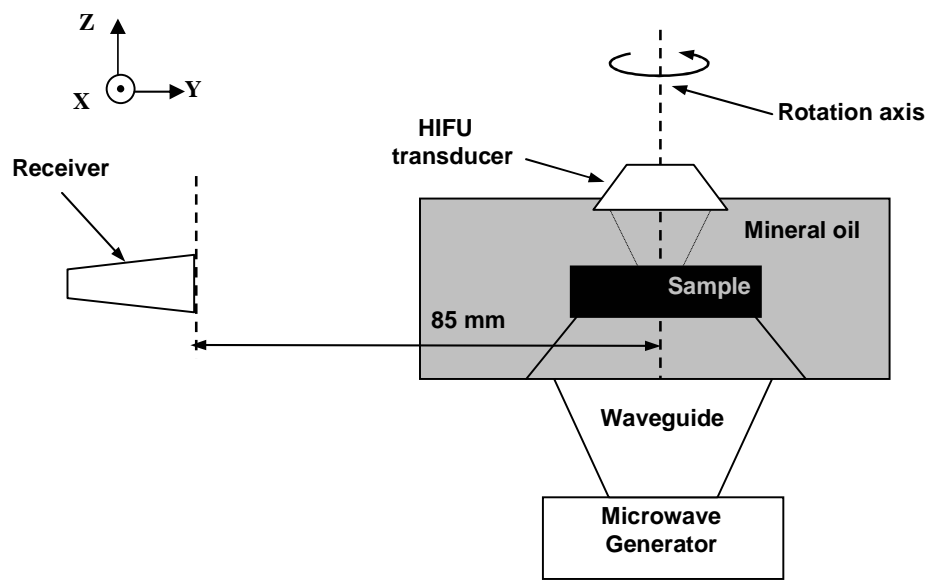

Figure 1: Side view of the experimental setup

An approximate 2-D filtered backprojection algorithm was proposed in [5]. To emphasize the sharp details in the reconstructed image, we used a local-tomography-type reconstruction algorithm to reconstruct the image [5]. 


\section{RESULTS}

Figure 2(a) shows the top view of the sample. Figure 2(b) gives a side view of the phantom used in the experiment. The lesion was created by the HIFU transducer at 18 watts for one and a half minutes. After heating, the tissue coagulated.

The lesion region lost some of its water content; therefore, its ability to absorb the microwave energy decreased accordingly. In our experiments, we carefully removed the air bubbles between the fat and muscle, and fixed their relative positions by a thin string. As we mentioned before, the penetration depth of the microwave in fat at $3 \mathrm{GHz}$ was about $9 \mathrm{~cm}$. Because the fat under the muscle was only about $1 \mathrm{~cm}$ in thickness, which is far below the penetration depth in fat, such a fat base had no obvious influence on the final results.

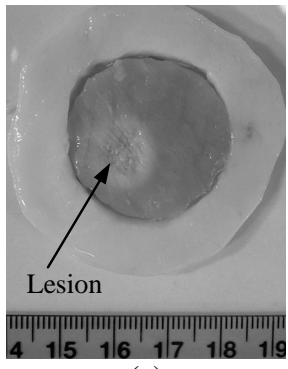

(a)

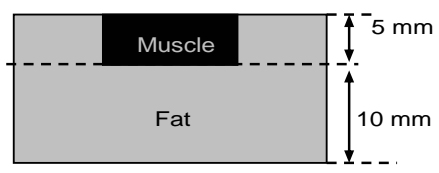

(b)

Figure 2: (a) Photograph of the phantom used in the experiment; (b) Side view of the phantom.

To better characterize the size and position of the lesion in the reconstructed image, we were interested in the interfaces between the muscle and the HIFU induced lesion, which were dominated by the high-frequency components of the thermoacoustic signals. We reconstructed the image using a local-tomography-type reconstruction algorithm. Figure 3(a) is the reconstructed image before we treated the sample; Figure 3(b) is the reconstructed image after the lesion was created. Those two images are not energy deposition images, because the low-frequency components of the thermoacoustic signals have been mostly removed to emphasize the boundaries. We observe a big change in the lesion region. The lesion shown in the sample photograph confirms the corresponding regions measured by TAT.

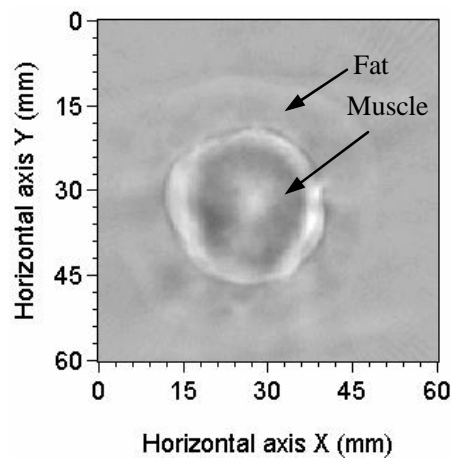

(a)

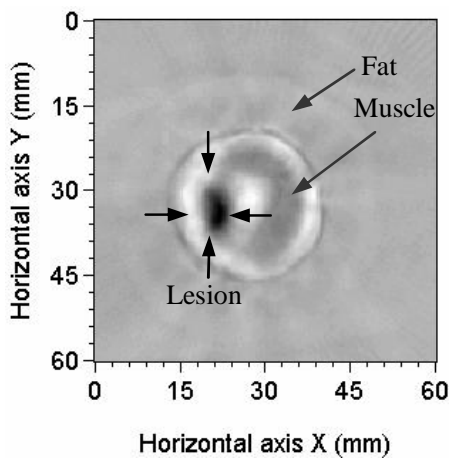

(b)

Figure 3: (a) Reconstructed image before heating; (b) Reconstructed image after heating. 


\section{CONCLUSIONS}

Tissue thermal-damage can result in a localized change in a tissue's electromagnetic properties, thus a change in its ability to absorb microwave energy. In our experiments, we have shown that HIFU-induced lesions can be assessed by TAT with good results. This imaging technology also has some distinct advantages over others. Compared with MRI imaging, our method is less expensive. Because of its small size, portable TAT imaging systems can be easily constructed. TAT is also compatible with HIFU treatment systems. Compared with B-mode ultrasound imaging, TAT has higher contrast, which is brought about by differences in the microwave absorption of different tissues; it has weaker speckle artifacts, because thermoacoustic signals recorded by the detector only propagate one-way in the tissue. Therefore, TAT has better image quality than B-mode ultrasound imaging. Compared with Elastography, our imaging technology can work on more heterogeneous tissues, such as breast cancer. Therefore, we conclude that thermoacoustic tomography has potential to become an effective, low-cost alternative for monitoring tissue thermal treatments.

\section{REFERENCE:}

1. Christopher J. Harvey, James M. Pilcher, et al, “Advances in ultrasound," Clinical Radiology, 57, pp157-177, 2002

2. Gail ter Haar, "Acoustic surgery," Physics Today, vol. 54, no. 12, 2001.

3. Francis A. Duck, Physical properties of tissue, a comprehensive reference book, Academic press, London, 1990

4. P. Stavroulakis ed., Biological effects of electromagnetic fields, Springer, 2003

5. Y. Xu and L.-H. Wang, "Reconstructions in limited-view thermoacoustic tomography", Med. Phys., vol. 31, no. 3, Mar., 2003

6. A. W. J. Dawkins, N.R.V.Nightingale, etc, "The role of water in microwave absorption by biological material with particular reference to microwave hazards", Phys. Med. Boil., vol.24, no. 6, pp1168-1176, 1979 